\title{
Design of an Innovative System for Wave Generation in Direct Tension-Compression Split Hopkinson Bar
}

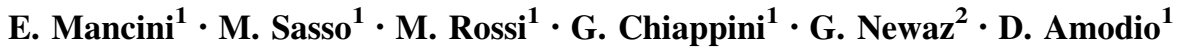

Received: 27 January 2015/Accepted: 11 April 2015/Published online: 23 April 2015

(C) Society for Experimental Mechanics, Inc 2015

\begin{abstract}
The split Hopkinson bar is a well-known apparatus for performing tests at high strain-rate on engineering materials. The specimen is placed between two long bars and quickly loaded by a pressure wave, which travels at sound speed along the input bar, passes through the specimen, then is partly reflected and partly transmitted to the output bar. In the classical version, the input wave is generated by the impact of a striker bar against the input bar at a given velocity. In the direct version of the Hopkinson bar, the wave is generated by pre-loading and releasing a portion of the input bar. However, with this approach the rise time of the pressure wave is usually longer respect to the classical version. In this paper, an innovative wave generation system is presented, which exploits the abrupt shear fracture of a thin brittle disc. In this way, the rise time of the incident waves can be close to
\end{abstract}

M. Sasso

m.sasso@univpm.it

E. Mancini

e.mancini@univpm.it

M. Rossi

m.rossi@univpm.it

G. Chiappini

g.chiappini@univpm.it

G. Newaz

gnewaz@eng.wayne.edu

D. Amodio

d.amodio@univpm.it

1 Dipartimento di Ingegneria Industriale e Scienze Matematiche, Università Politecnica delle Marche, Via Brecce Bianche, Ancona, Italy

2 Department of Mechanical Engineering, Wayne State University, 5050 Anthony Wayne Drive, Detroit, MI 48202, USA that obtained by the classical impact-based Hopkison bars ( $80 \mu \mathrm{s}$ instead of $50 \mu \mathrm{s}$ ). Moreover, both tension and compression tests can be carried out with the same experimental system.

Keywords Split Hopkinson tension-compression bar . High strain rate $\cdot$ Metals

\section{Introduction}

The characterization of materials at different strain rates is an important aspect for proper design, verification, and numerical simulation of mechanical components subjected to dynamic loads. The Hopkinson bar [1-3] is probably the most common apparatus for testing at high strain-rate. During a Hopkinson bar experiment, a small specimen is sandwiched between two long bars and is ideally subjected to a uni-axial compressive or tensile load at high strain rate. This method, initially developed for compression tests [2] but later extended to tensile tests [3, 4] and torsion [5], is today the most used to carry out tests in the range of strain rates ranging from $10^{2}$ to $10^{4} \mathrm{~s}^{-1}$. These tests are mainly used to characterize the constitutive behavior of materials, using one of the models available in the literature for ratedependent materials. This is an essential step to perform finite element simulations of various dynamic phenomena (shocks, ballistics, etc.).

In its classical version, generally named split Hopkinson pressure bar (SHPB), a compressive load is generated by the impact between a projectile, or striker bar, onto an incident bar. In order to obtain a tensile load, the compressive wave must be reversed to a tensile one before it reaches the specimen. Different solutions have been 
proposed in the literature to overcome this problem: Lindholm and Campbel [6] designed suitably shaped samples that allow direct use of the compression wave to achieve tension. Other researchers adopted a collar, as done by Nicholas [7] or embedded the specimen into the bars, as done by Bragoc and Lomunov [8], to protect it from the compressive wave. In this case, the free end of the output bar is exploited to invert the load sign. Later, different modifications to SHPB were developed in order to generate directly a tension wave in the input bar. Recently, this loading method has become more and more desirable because it allows the optical access to the specimen surface, facilitating the application of high speed imaging to tensile tests. One of the most popular version for tensile tests, commonly named split Hopkinson tension bar (SHTB), consists in a tube-like impactor that is placed around the input bar and is accelerated by compressed air towards an anvil at the outer end of the input bar [9-14]. Hence, a tensile wave is generated and propagates along the input bar towards the specimen. Adjusting the gas pressure can regulate the stress pulse magnitude. However, with this method, the loading bar remains unsupported along the entire length between impact flange and the beginning of the striker tube. This prevents from using long tubes and consequently determines short input waves. A striker bar with non-circular cross section has been proposed by Gerlach et al. [15] to overcome this limitation. They designed an U-shaped striker that, surrounding the input bar without being in contact with it, allows to generate clean stress pulses with a duration of about $1 \mathrm{~ms}$.

The methods described above often require complex setup for the SHTB. Easier SHTB designs are those based on pre-stressing a portion of the incident bar, as implemented in [4, 16-20]. By loading the pre-stressed bar in tension an elastic energy is stored. A brittle intermediate piece (named sacrificial element in the rest of the paper) is placed between the pre-stressed and the incident bars, it is broken, and a tensile stress wave is generated. This solution has been used by Staab and Gilat [4] to investigate the effect of specimen geometry, and by Quick et al. [21] to investigate the behavior of thin sheet metals under different deformation modes. Tarigopula et al. [22] assessed the performance of digital image correlation technique in high strainrate tests. Among the papers that addressed the issue of generating direct tension waves, a solution based on friction clamping lock/unlock appeared too. This method, by clamping part of the input bar, avoids movements during the pretension as described by Chen et al. [23]. Unfortunately, with this SHTB design it may be difficult to generate a continuously rising stress pulse. Furthermore, it is impossible to adopt the pulse shaping technique based on dummy-specimen [15, 23].
In this paper, a new split-Hopkinson tension compression bar (SHTCB) is described. This system overcomes the issues described so far and performs both tension and compression tests with the same set-up. An innovative tension-compression wave generation system, based on the static shear failure of a thin brittle disc, is reported. The new system is able to handle both tasks: preloading and breaking of the sacrificial element. So, the sign of the incident wave can be changed either by inverting the direction of the actuation load or by swapping the position of the sacrificial disc, i.e. upstream or downstream with respect to the pre-stressed bar. The acquisition sensors and cablings are identical for tension and compression tests. The stress wave magnitude can be adjusted by changing the thickness of brittle disc. A pulse duration longer than $1.1 \mathrm{~ms}$ is obtained. This allows to test highly ductile materials and to perform tests at lower strain rates (about $2 \times 10^{2} \mathrm{~s}^{-1}$ ). Moreover, the system produces rise times of the incident wave, close to those obtained with the classical version. If longer time ramps are desired, the particular set up of the temporary blocking system also permits the use of pulse shapers. Finally, to validate the quality of the new designed SHTCB, the experimental activity, carried out on tensile and compression tests, is reported in terms of stressstrain curves at different strain rates.

\section{Theoretical Background}

The split Hopkinson pressure bar is probably the most used apparatus to carry out tests at high strain rates, ranging from $10^{2}$ to $10^{4} \mathrm{~s}^{-1}$. It is made by three aligned bars: the first, named striker bar, is fired against the so called input bar. The latter is separated from the output bar by the specimen that is sandwiched between them. When the striker bar impacts the input one, a uniaxial elastic stress wave is generated and propagates along the first (incident) bar. When the incident wave reaches the specimen, because of the impedance mismatch between the bar and the specimen, a part of it is reflected back along the input bar while the rest travels through the specimen and enters the second (output) bar. Meanwhile, several stress wave reflections occur within the relatively short specimen (Davies and Hunter [24]) rapidly leading to a state of quasi-static equilibrium. The bars are designed to stay within the elastic limit, while the sample deforms plastically.

The waves that propagate into the bars are measured using two strain gauge rosettes appropriately placed, which convert the stress waves into proportional analog signals. The mechanical behavior of the sample material can be evaluated if some simplifying hypotheses are assumed: uniaxial stress in the sample, negligible specimen length, negligible friction effect, and uniaxial wave propagation. 
Leaving aside the detailed discussion, it is possible to obtain the displacements $u_{1}$ and $u_{2}$ and the loads $P_{1}$ and $P_{2}$ at the lateral faces of the sample (subscripts 1 and 2 indicate the interface of the left and right side respectively):

$$
\begin{aligned}
u_{1}(t) & =C_{0} \cdot \int_{0}^{t}\left[\varepsilon_{r}(\tau)-\varepsilon_{i}(\tau)\right] \cdot d \tau, u_{2}(t) \\
& =-C_{0} \cdot \int_{0}^{t} \varepsilon_{t}(\tau) \cdot d \tau, \\
P_{1}(t) & =A_{b} E_{b}\left[\varepsilon_{i}(t)+\varepsilon_{r}(t)\right], P_{2}(t)=A_{b} E_{b} \varepsilon_{t}(t)
\end{aligned}
$$

where $C_{0}, E_{b}$ and $A_{b}$ indicate respectively the sound speed, the elastic modulus and the cross section area of the bars, which are supposed to be identical. The incident strain wave generated upon impact is represented $\varepsilon_{i}, \varepsilon_{r}$ is the reflected one and $\varepsilon_{t}$ is the transmitted one. If the sample deforms uniformly and is in dynamic equilibrium, i.e. $P_{1}(t)=P_{2}(t)$ and $\varepsilon_{i}(t)+\varepsilon_{r}(t)=\varepsilon_{t}(t)$, than the engineering stress, strain rate and strain experimented by the sample can be obtained from the classical "reduced" formulae:

$$
\begin{aligned}
& \dot{\varepsilon}(t)=-\frac{2 C_{0}}{L_{s}} \varepsilon_{r}(t) \\
& \varepsilon(t)=-\frac{2 C_{0}}{L_{s}} \int_{0}^{t} \varepsilon_{r}(t) \cdot d t \\
& \sigma(t)=\frac{A_{b} \cdot E_{b}}{A_{s}} \varepsilon_{t}(t)
\end{aligned}
$$

where $L_{\mathrm{S}}$ and $A_{\mathrm{S}}$ represent respectively the initial length and cross-sectional area of the sample. Excluding time from previous Eqs. (3-5) i.e., synchronizing the reflected and transmitted signals, the flow stress-strain law of the material at high strain rate is obtained.

This classic version is only capable to generate compression waves and hence to perform only compression tests. In order to carry out tensile tests it is necessary that the input wave reaches the free end of the output bar without deforming the specimen (e.g. by an external collar as done by Nicholas [7]), then it is reflected as a tensile wave, which will load the sample in tension.

\section{Direct Split Hopkinson Bar Apparatus}

The apparatus for direct version of split Hopkinson bar is more compact, since the launch system of the striker bar is not present. This allows the generation of longer waves at equal total size. Furthermore, the specimen is loaded directly by the first generated wave and no collar is needed to connect the input and output bars. A possible limitation of this set-up is that the stress input wave has an intensity equal to half of the statically applied pre-tension stress. Moreover, particular attention is needed for the release system since its effectiveness and rapidity strongly affects the shape and the raising time of the input wave. Similarly to the classic SHB, the pulse duration can be changed by varying the length of the pre-stressed bar (that replaces the striker bar).

In the direct version, the wave is generated by preloading a portion of the input bar (named pre-stressed bar) and by the subsequent instantaneous release of this load. However, the principle to evaluate the flow stressstrain curve of the tested material is the same explained in "Theoretical Background" section, as described in different papers [4, 16-23].

The preload is applied by means of an actuator at the extremity of the pre-stressed bar. A rigid block is used to contrast the preload at a given distance. The load may be released either at the actuator/bar interface (upstream) or at the bar/block interface (downstream). If the load is released at the pre-stress bar/actuator interface, the generated wave is of opposite sign to the preload, i.e. a preload in compression will generate a tensile wave (Fig. 1a) whereas a preload in tension will generate a compression wave (Fig. 1c). Conversely, if the load is released at the block/ input bar interface, the generated wave is of the same sign to the preload (see Fig. 1b and d).

The traveling wave has half intensity but double spatial length with respect to the pre-stressed bar.

The release of the preload may be accomplished by a fast unclamping at one extremity of the pre-stressed bar as done in $[4,20,22,23]$ or by failure of a sacrificial element $[19,21]$.

\section{Developed Apparatus (SHTCB)}

In this work, the entire apparatus was specifically designed to perform, with a unique equipment, compression and tension tests on ductile materials from hundred to few thousands of strain rate $\left[\mathrm{s}^{-1}\right]$. As such, long pulses are needed, and all the available length in laboratory should be exploited at best. For this reasons the direct version of the SHB has been chosen. The apparatus is made of 17-4 PH bars, all having the same diameter $(18 \mathrm{~mm})$. The input bar is $7.5 \mathrm{~m}$ in length, the output bar $4.0 \mathrm{~m}$. A $3 \mathrm{~m}$ long bar is pre-stressed in tension or compression by means of an electro-mechanical actuator with a $100 \mathrm{kN}$ maximum load. A $6 \mathrm{~m}$ long input wave, corresponding to $1170 \mu \mathrm{s}$ in time, is generated. Evenly spaced polymeric 
(a) Tension test: Pre-stressed in compression, upstream release

Release

this part

Blocking system

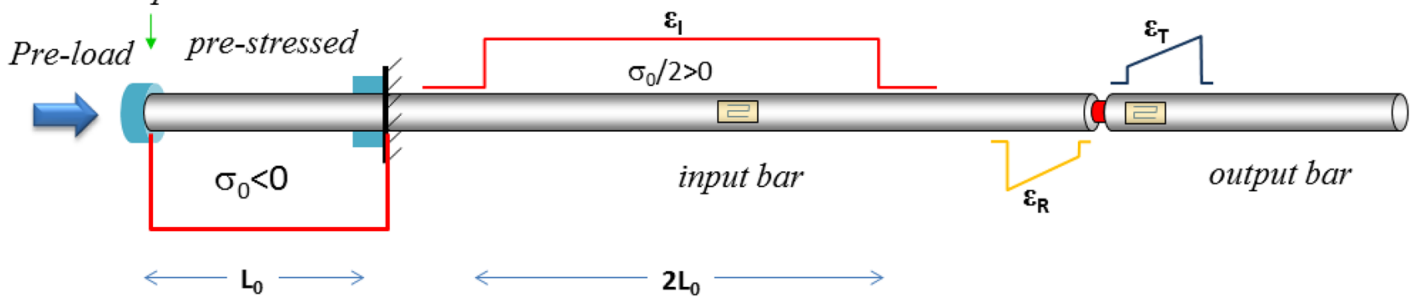

(b) Tension test: Pre-stressed in tension, downstream release

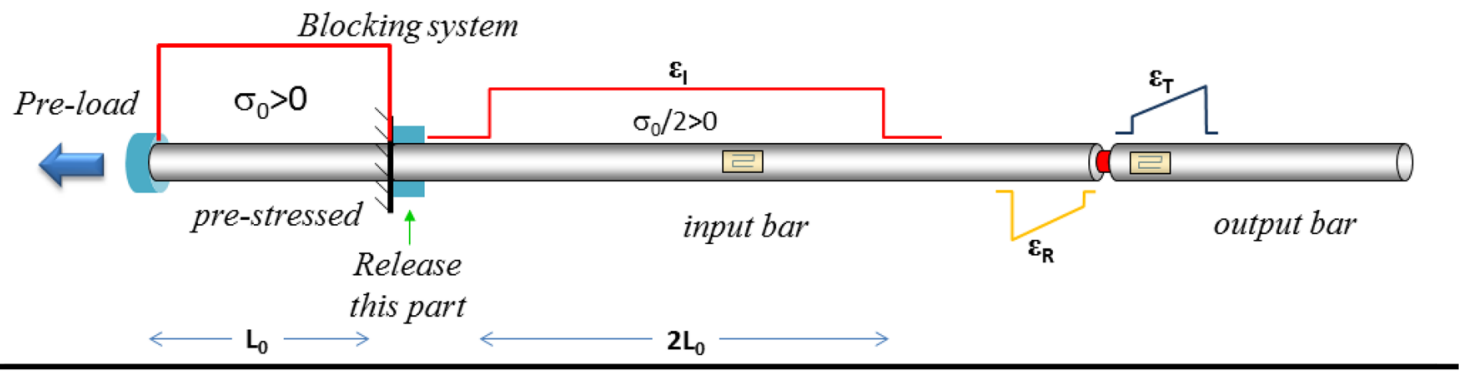

(c) Compression test: Pre-stressed in tension, upstream release

Blocking system

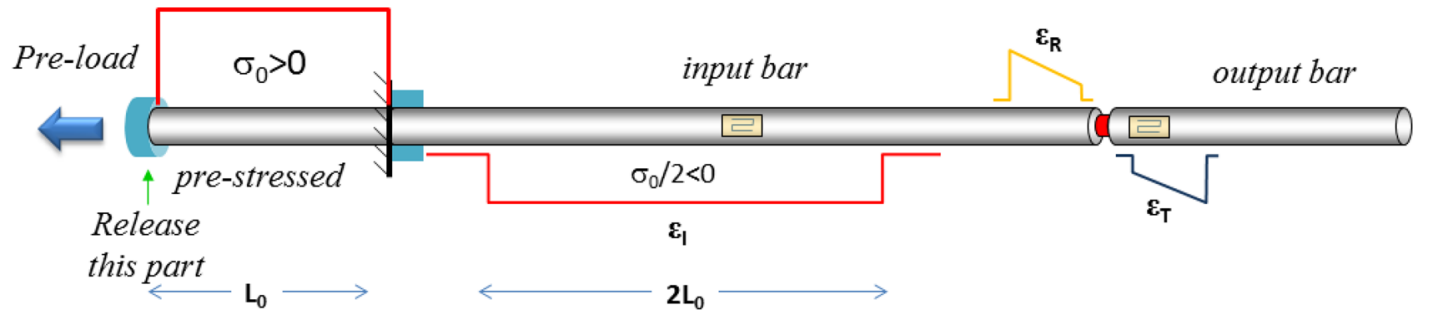

(d) Compression test: Pre-stressed in compression, downstream release

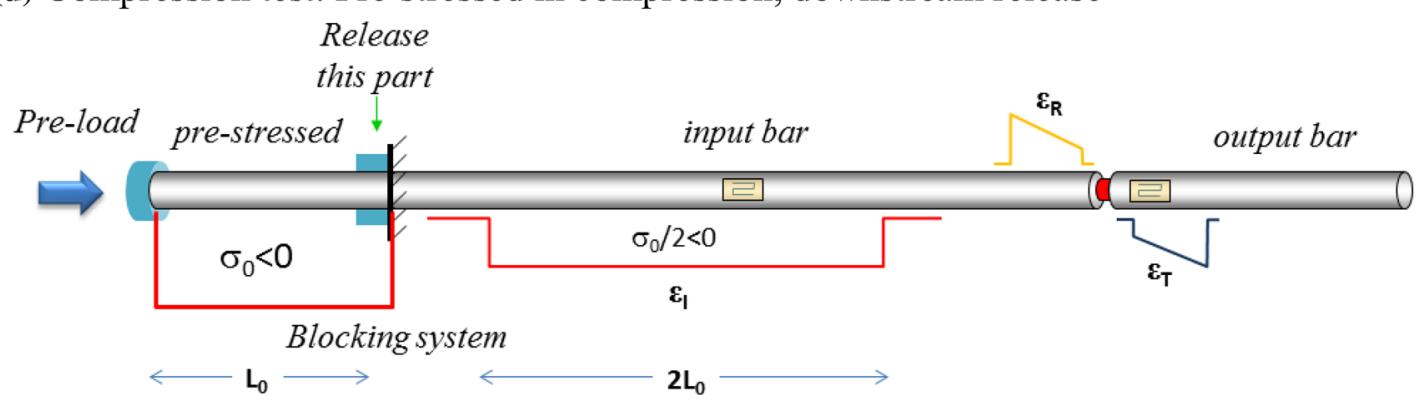

Fig. 1 Wave generation: schematic representation of direct tension-compression bar

supports are placed to sustain the input and output bars at $1 \mathrm{~m}$ one from each other. On the other hand, 11 supports are used for the pre-stressed bar to prevent compression buckling.

Pictures of the realized SHTCB are shown in Fig. 2. The HEA support beams were painted in different colors to better distinguish the different parts of the system: green for the pre-stressed bar, white and red for the input and output bars.

\section{Signal Acquisition System}

Strain gauges are installed at half span of the input bar and at the beginning of output bar for measuring the input, reflected and transmitted pulses. Also the pre-stressed bar is instrumented with a strain gauge in order to monitor the preload in real time. The full bridges configuration was chosen to avoid spurious flexural and thermal contributions to the measurements. Prewired tee rosettes (model Vishay ${ }^{\circledR}$ 


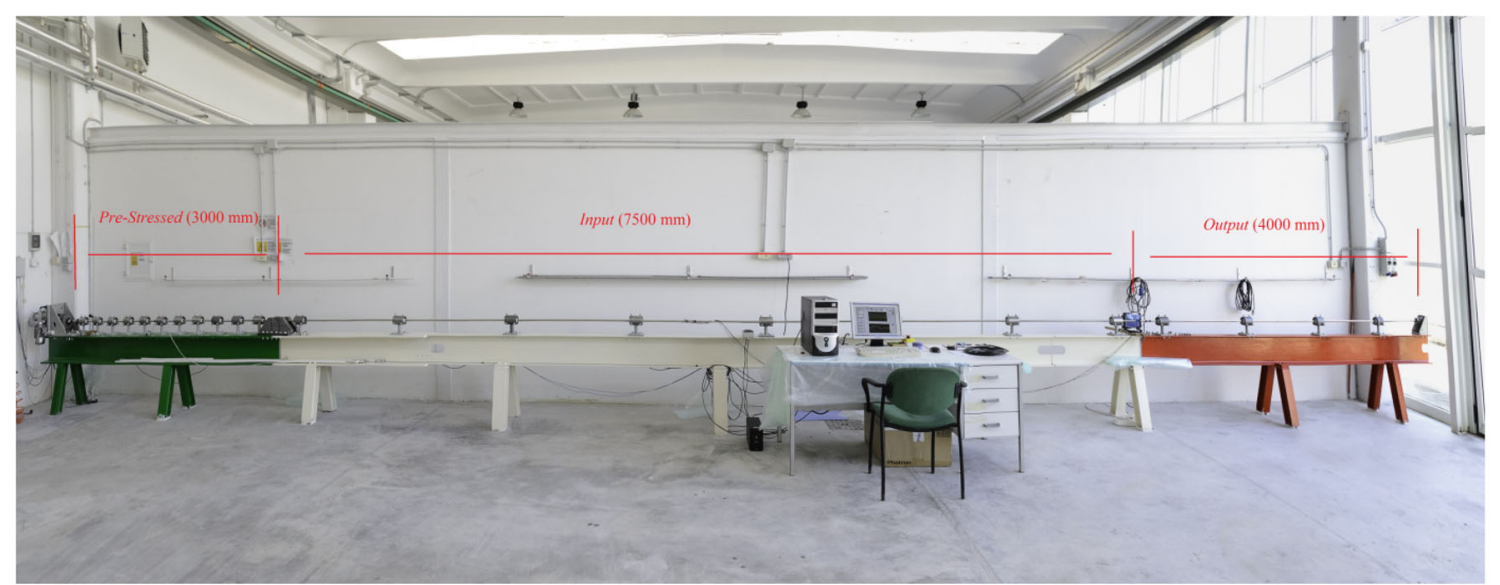

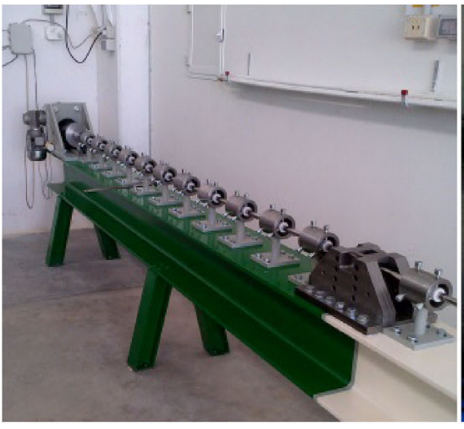

(a) Pre-Stressed (3000 mm)

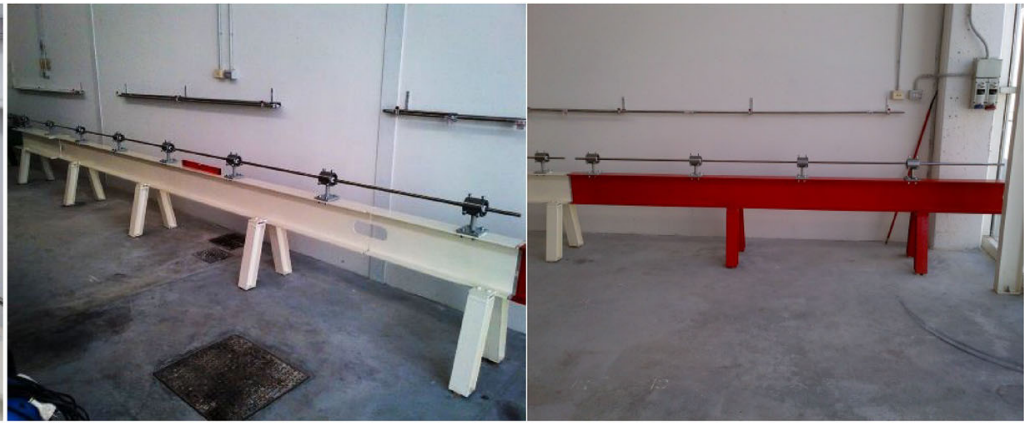

(b) Input (7500 mm) (c) Output (4000 mm)

Fig. 2 Direct tension-compression split Hopkinson bar

C2A-62LT) were used with a small gauge length $(1.57 \mathrm{~mm})$ to avoid any unintended low pass filter effect. Moreover, the prewired solution has been preferred to avoid "onboard" welds. The Wheatstone bridges are power supplied by standard DC batteries operating at $9 \mathrm{~V}$. The signals are acquired by a data acquisition card $\left(\mathrm{NI}^{\circledR}\right.$ PCI 6120 ) operating at $1 \mathrm{MHz}$ sampling rate on 4 simultaneous channels. The 16 bit analog-digital converter is used on a $200 \mathrm{mV}$ full-scale range, resulting in a voltage resolution of $6 \mu \mathrm{V}$. With this value the amplification was considered not necessary. The electronic equipment is also able to trigger the acquisition of a high-speed camera that could be useful to observe the sample deformation during time.

\section{New Wave Generation System}

In the direct version two important mechanical components must be analyzed: the pre-loading system and the blocking system. In particular, the former is critical because it should provide a sudden release of the bar to obtain a short rise time, analogous to that of the classic version. If the sacrificial element is not brittle enough or excessive additional mass is connected at the bar/actuator interface, the raise time will increase dramatically.
Also the blocking system at the end of the pre-stressed bar is very important, and should be as rigid as possible. Indeed, a compliant constraint would cause an interaction between the blocking system and the bars, determining an irregular shape of the input wave. The newly developed system described in this paper effectively solves both critical problems.

The static pre-stress load is provided by means an electro-mechanical actuator at the left most extremity. It is important to highlight that the fixture connecting the actuator to the pre-stressed bar accomplishes the double task of transmitting the preload and breaking the sacrificial element. So, no further tools or hydraulic jacks are needed for releasing the load. Furthermore, the wave generation system is designed such that tension or compression tests can be carried out by just inverting the direction of actuation force.

\section{Upstream Rupture}

The force of the electro-mechanical actuator is transmitted to the pre-stressed bar by means of a thin sacrificial disc loaded in shear. The disc is made of $55 \mathrm{NiCrMoV} 7$ tool steel, which presents a failure stress of approximately $2000 \mathrm{MPa}$ after quenching with very small plastic 


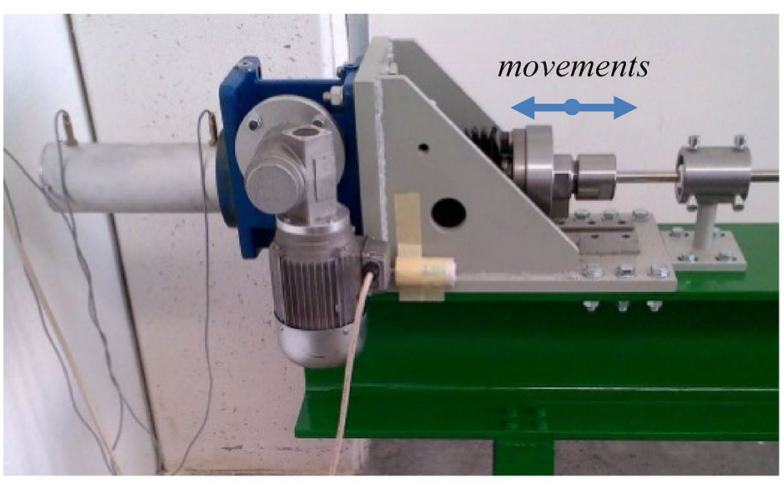

(a) Component

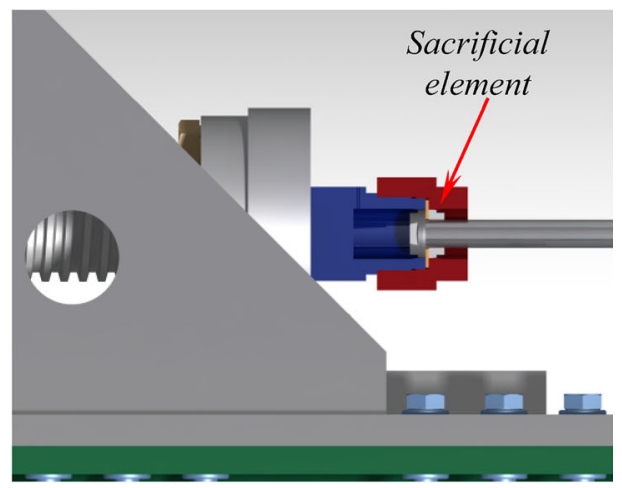

(b) CAD view

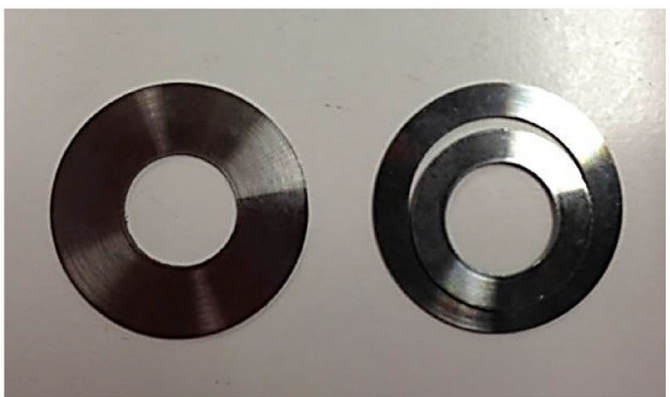

(c) Sacrificial disc element

Fig. 3 a Pre-stressed bar system, b upstream rupture system and c sacrificial disc element: (before and after test)

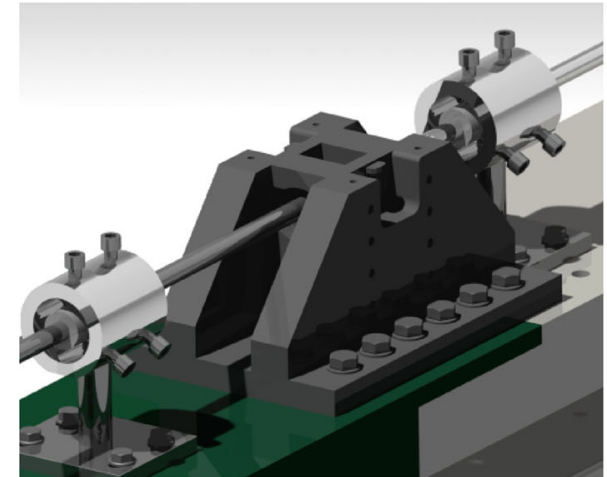

(a)

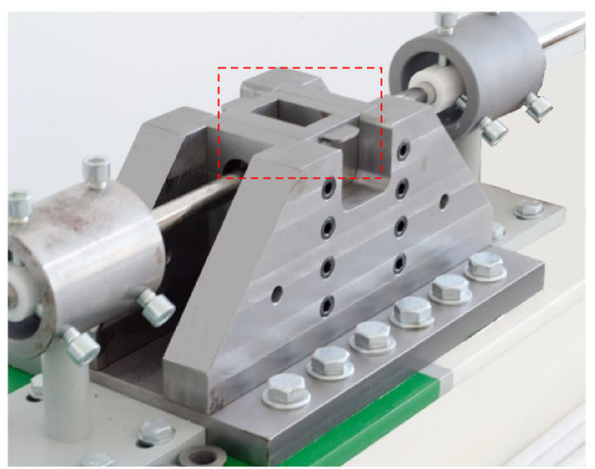

(c)

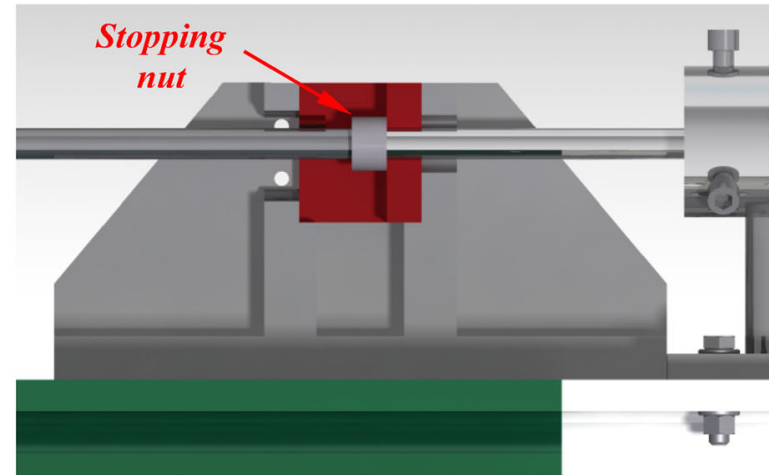

CAD

(b)

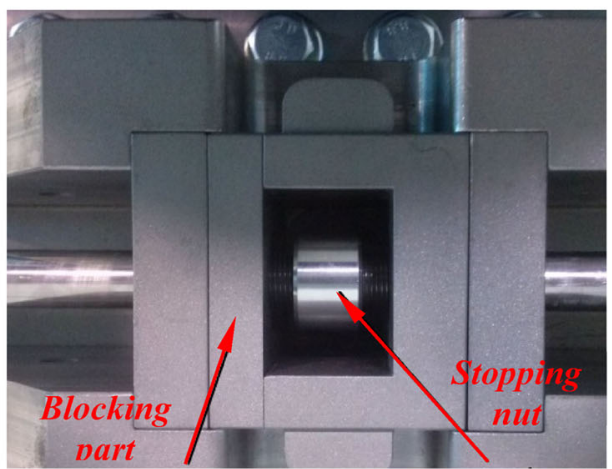

(d)

Fig. 4 Blocking system: 3D CAD view $(\mathbf{a}, \mathbf{b})$ and real component pictures $(\mathbf{c}, \mathbf{d})$ 
Fig. 5 Positioning of the eventual pulse shaper

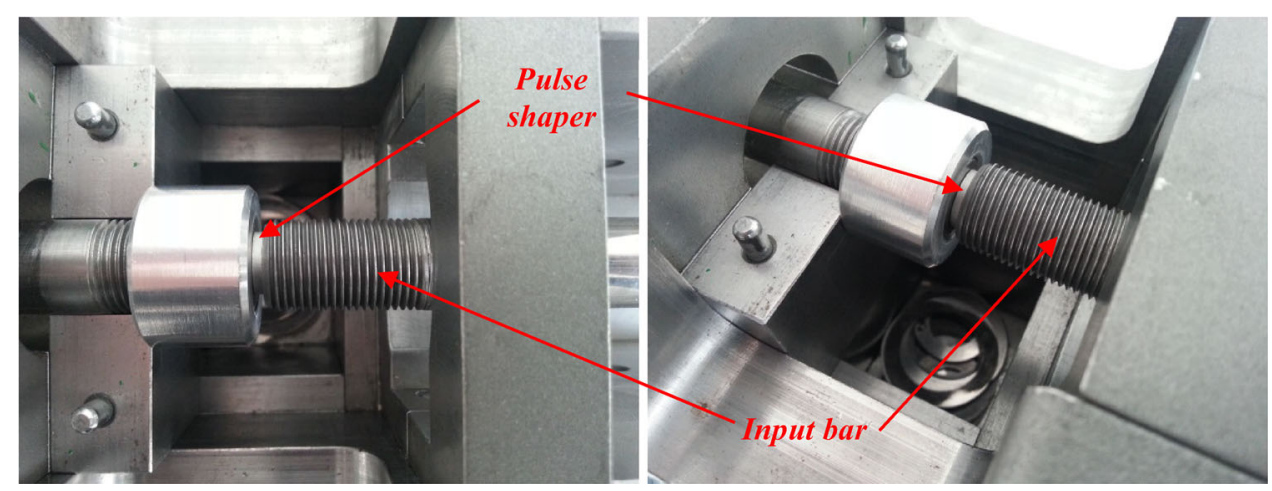

Fig. 6 End arrest: a 3D CAD view, b real component

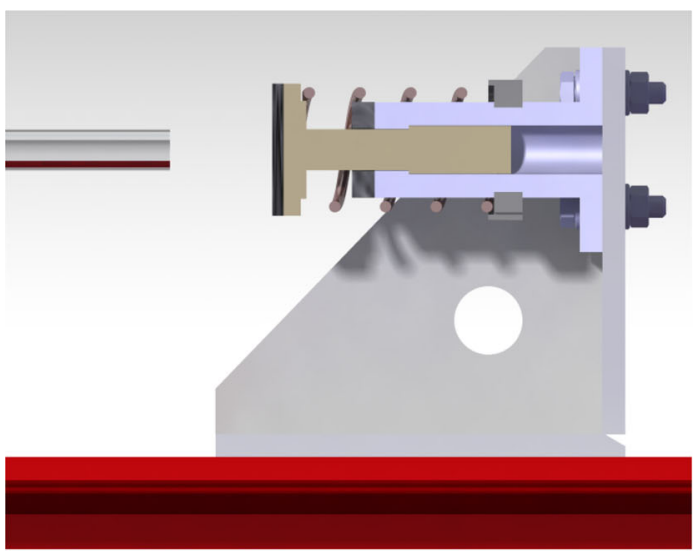

(a)

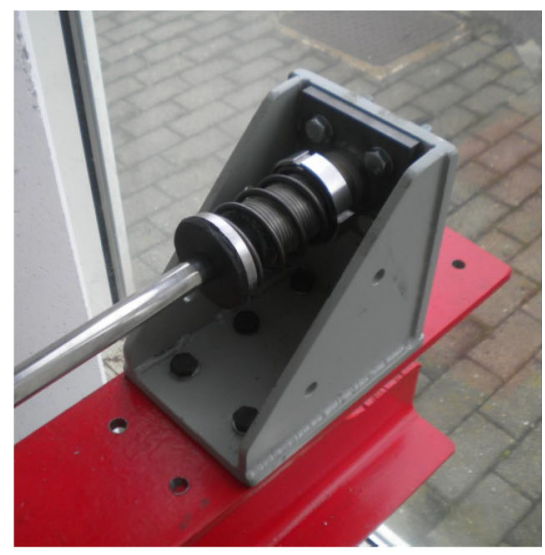

(b)

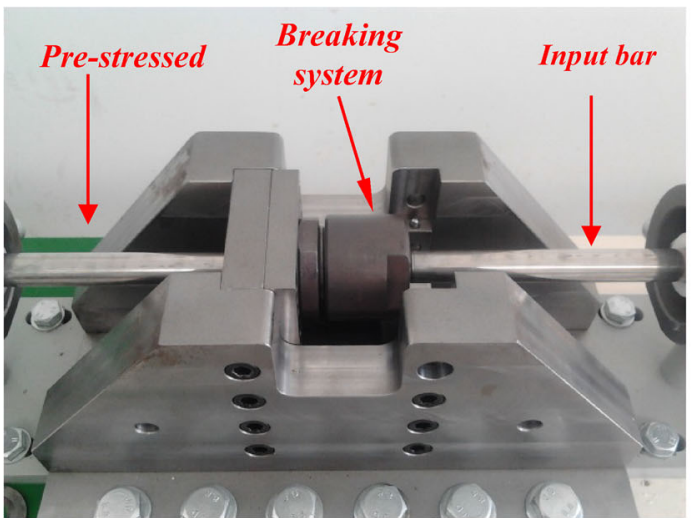

(a) Component

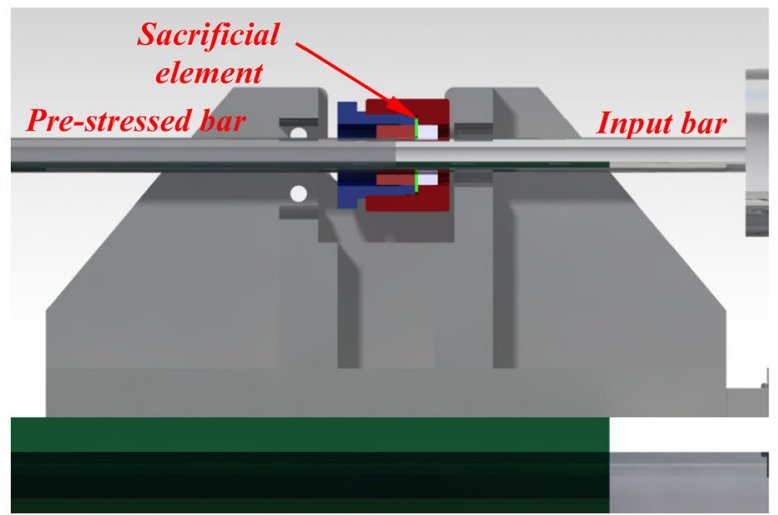

(b) $\mathrm{CAD}$ view

Fig. 7 Downstream breaking system

deformation. The high strength permits to hold up to $100 \mathrm{kN}$ pre-load with only $0.7 \mathrm{~mm}$ thickness. The brittleness of the material and the small thickness of the discenables an extremely fast load release, on the order of few tenths of microseconds.

Three meters away from the actuated extremity, the prestressed bar is connected to the input bar by means of screwed nuts. The same nuts are used to stop the pre-stress bar against a rigid block with a unilateral contact. The sudden shear rupture of the sacrificial disc, caused by two cylindrical nuts with sharp edges, generates a release wave that travels towards the rigid block and continues into the input bar. As said, the generated input wave is twice the pre-stressed bar in length, with a stress intensity that is half of the pre-load. Since the sacrificial element failure occurs in shear, the load direction can be inverted without any 


\section{Compression waves}

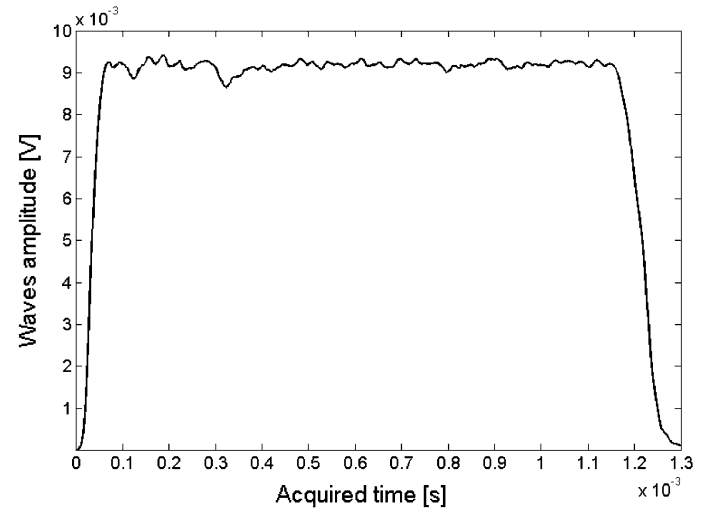

(a) upstream rupture

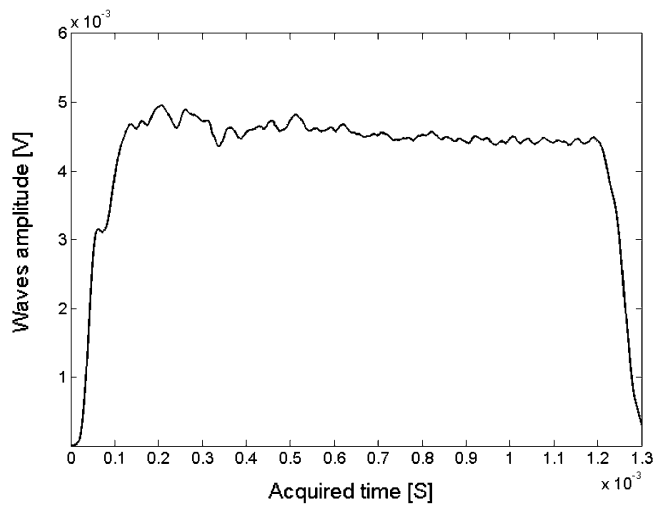

(b) downstream rupture (annealed disc)

Tension waves

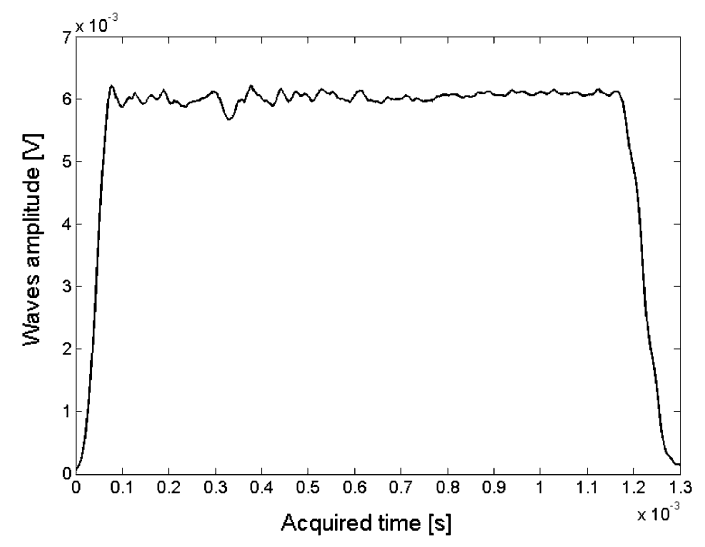

(c) upstream rupture

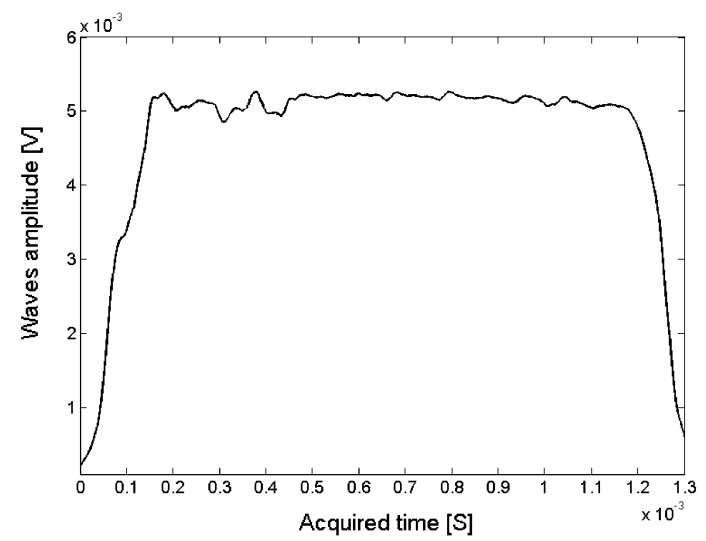

(d) downstream rupture (annealed disc)

Fig. 8 Tension and compression incident waves measured with the present SHTCB

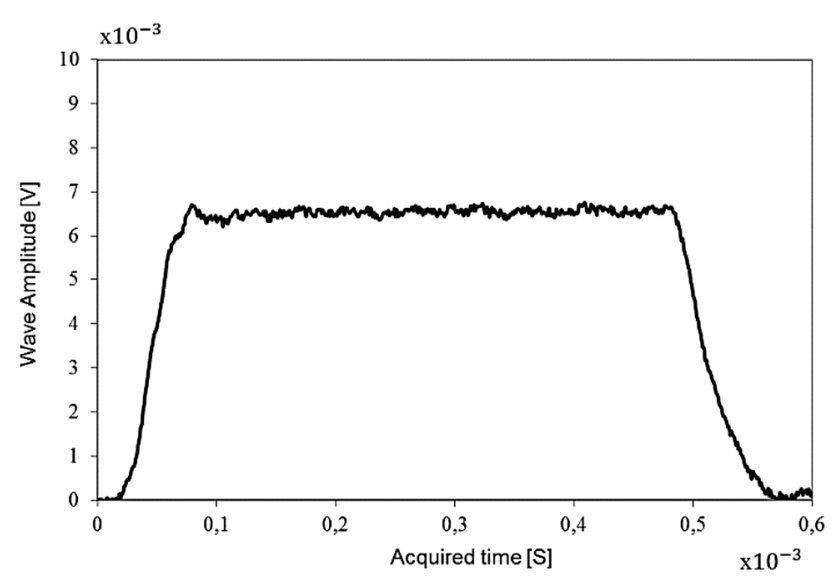

Fig. 9 Compression wave obtained with the classical SHPB

modification of the loading system. Only the current polarity of the electrical motor has to be inverted. Furthermore the blocking system is the same for tension and

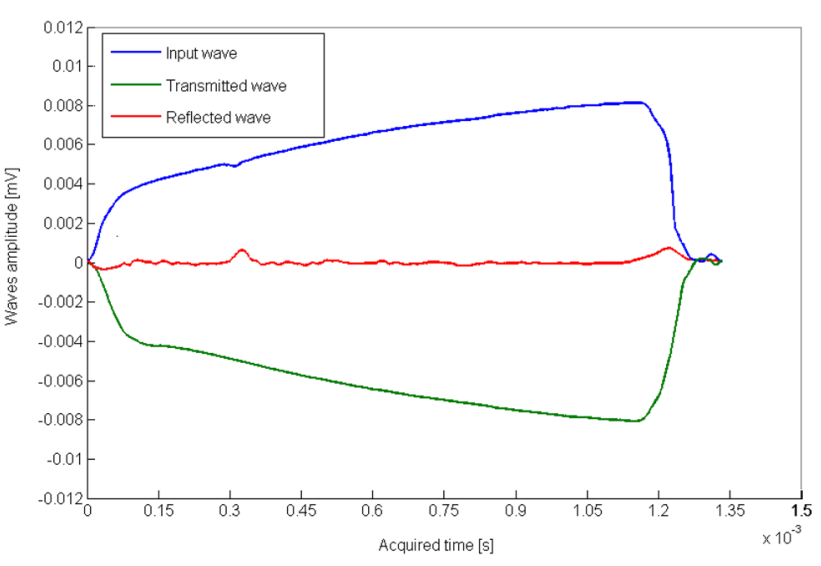

Fig. 10 Pulse shaper effect

compression, the only difference is given by the nut-block contact occurring at opposite face. Pictures of the loading and release systems are reported in Fig. 3, together with an 


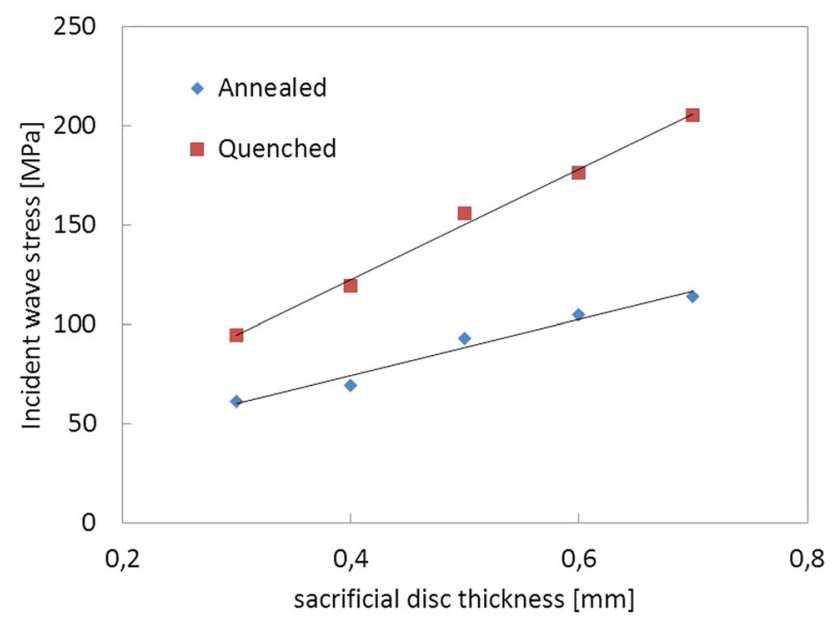

Fig. 11 Sacrificial disc thickness effect

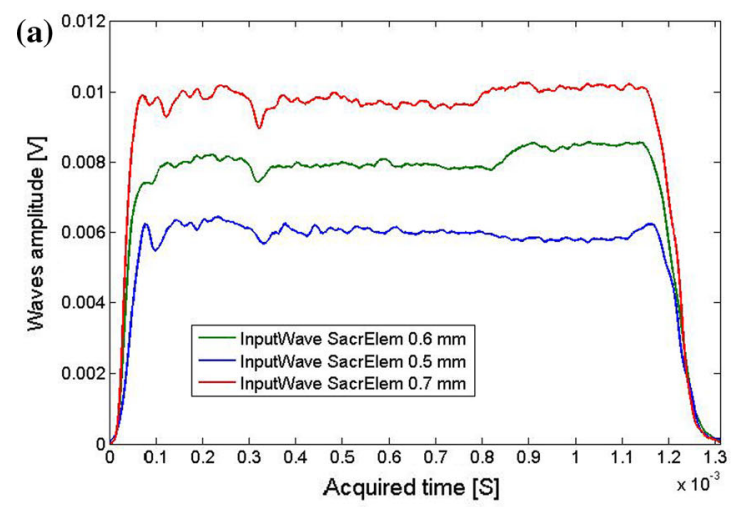

example of sacrificial disc (Fig. 3c). The nuts and blocking system are shown in Fig. 4.

The stopping nuts technique may allow for pulse shaping when necessary, by interposing a dummy disc (usually made of copper or aluminum) between the pre-stressed and the input bar, as shown in Fig. 5. In this case the prestressed and the input bars are not connected by the stopping nut. On the contrary, they sandwich the pulse shaper with a small preload provided by the spring of the end arrest.

A terminal block, named the end arrest, is placed at the end of the output bar to stop the compressive wave in compression tests (Fig. 6).

The end arrest is also used to sandwich the specimen between the input and output bars by means of an

Fig. 12 Acquired waves (a) and rise time (b)

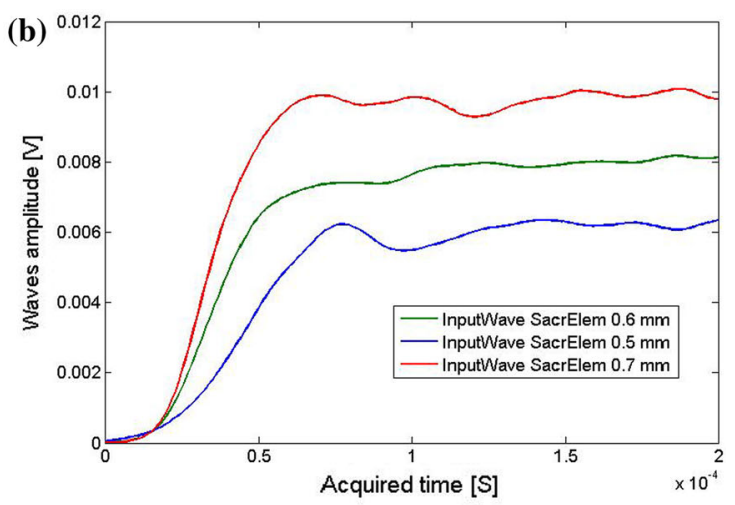

Table 1 Comparison between classic to direct split Hopkinson bar

\begin{tabular}{lll}
\hline & SHPB @ WSU & SHTCB @ UPM \\
\hline Geometry \& material & 4340 steel & $17-4$ PH steel \\
Elastic modulus $(\mathrm{MPa})$ & & \\
Density $(\mathrm{kg} / \mathrm{mc})$ & 205,000 & 204,000 \\
Sound speed $(\mathrm{m} / \mathrm{s})$ & 7850 & 7800 \\
Striker bar length $(\mathrm{mm})$ & 5110.2 & 5114.1 \\
Input bar length $(\mathrm{mm})$ & 1100 & 3000 \\
Output bar length $(\mathrm{mm})$ & 3073 & 7500 \\
Diameter (mm) & 3073 & 4000 \\
Typ. spec. length $(\mathrm{mm})$ & 12.7 & 18 \\
Upper limit & 4 & 6 \\
Max incident wave stress $(\mathrm{MPa})$ & & 200 \\
Max strain rate $\left(\mathrm{s}^{-1}\right)$ & 200 & 1671.2 \\
Max strain $(\%)$ & 2492.8 & 196 \\
Lower limit & 75 & 1173.2 \\
Pulse duration $(\mu \mathrm{s})$ & & 170.0 \\
Min strain rate for failure@ $20 \%\left(\mathrm{~s}^{-1}\right)$ & 430.5 & \\
\hline
\end{tabular}


adjustable spring. This provide a small but sufficient friction force to maintain the specimen in the correct position during the pre-loading. On the other hand, in tensile tests, a slightly modified configuration of the end arrest provides a small pre-tension on the specimen and avoids any gap in the threaded connection between bars and specimen itself.

\section{Downstream Rupture}

Although the upstream rupture configuration have been successfully used for performing tension and compression tests, as will be shown in "Experimental Wavegeneration Verification" section, the blocking system of Fig. 4 has been designed in a modular way, so that it can be mounted to permit the installation of the sacrificial disc. This corresponds to the version of direct SHTB that is more commonly encountered in literature [4, 20, 22, 23].

A picture of the system is shown in Fig. 7a. Figure 7b shows a section view of the system and highlights the location of the sacrificial element. If the pre-stress is in tension the blue fixture will be in contact with the rigid block. On the contrary, the red fixture will stop the bars when the pre-stress is in compression. Again, the load release occurs when the brittle disc fails in shear, then the connecting nuts will slide inside the outer fixtures and the pulse will start traveling into the input bar.

\section{Experimental Wavegeneration Verification}

To evaluate the quality of the generated waves in terms of rise times and shape, the pressure waves are compared with the one obtained using the classical SHPB with a striker impact speed of $10 \mathrm{~ms}^{-1}$. The tests have been carried out without the sample, both pre-tensioning and pre-compressing up to 10 tons. The typical input waves obtained in

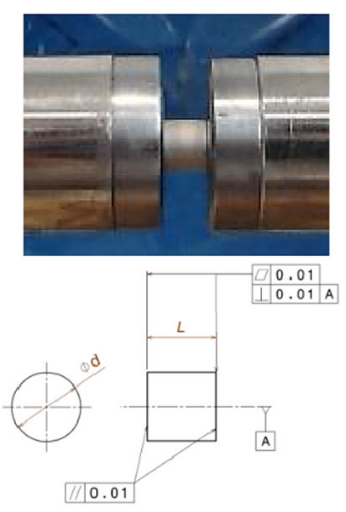

(a) Compression

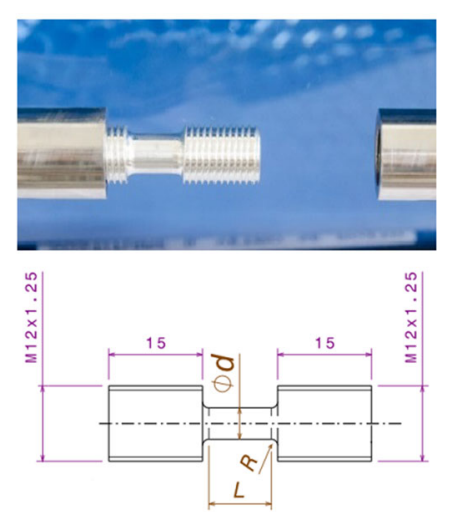

(b)Tension
Fig. 13 Specimens geometry the different configuration are shown in Fig. 8. Figure 9 shows the incident wave generated by the impact of the striker bar.

It is observed that the shape of the waves are very similar, all of them are close to the theoretical rectangular wave-form. Some fluctuations are present in all cases, including the classical SHPB, which are probably due to small of misalignments the bar or friction/contact interactions with supports. These effects are not specifically related to the present Hopkinson bar design. Finally, the rise time are not so different, varying from the $50 \mu$ s measured in the impact version of the SHPB to the $60-80 \mu$ s for the present one. Slightly longer rise times, about $120 \mu$ s, are achieved with annealed discs. The effect of a soft aluminum pulse shaper is shown in Fig. 10, where the oscillations are eliminated and a rising input wave is obtained. This may be desirable for achieving constant strain rate during compression test.

At the end, the effect of the sacrificial disc thickness has been evaluated, obtaining an almost linear relation with the input wave amplitude, as shown in Fig. 11. The resulting incident waves obtained with three different thicknesses are shown in Fig. 12a. Figure 12b shows an enlargement of the rising phase of the waves.

\section{Comparison of Classic Versus Direct SHB}

Table 1 shows a comparison, in term of the values of minimum and maximum obtainable strain rate, between the classic version (installed at the Mechanical Engineering Department of Wayne State University [25]) and the newly built direct tension-compression one. Obviously, a long pulse and a long specimen are preferable for lowering the strain rate towards intermediate values. The lower limit for the strain rate, reported in Table 1, is related to the hypothesis of $20 \%$ strain to failure. Then, the minimum strain rate achievable is simply given by $\dot{\varepsilon}_{\min }=0.2 / \Delta t$, with $\Delta t$ representing the pulse duration. On the other hand, shorter specimens lead to higher strain rates so, the maximum attainable strain rate is higher in Hopkinson bars with smaller diameter. The ideal maximum strain and strain rate values reported in Table 1 are for specimens of typical length, i.e. 1/3 of the bar diameter as suggested by Nicholas [7] assuming that all the incident energy is reflected, i.e. the specimen has negligible mechanical strength. The upper limit of the incident wave stress in the classical version is related to the maximum impact speed provided by the air gun. In the proposed SHTCB version, the M18 threaded connections in the pre-stressed bar limits the admissible static stress to roughly $400 \mathrm{MPa}$, corresponding to $200 \mathrm{MPa}$ for the travelling wave. 
Table 2 Compression tests data

\begin{tabular}{llllll}
\hline ID\# & D & L & Disc thickness & avg. eng. stain rate & avg. log strain rate \\
\hline 1 & 5 & 5 & 0.3 & 140 & 150 \\
2 & 8 & 5 & 0.6 & 300 & 360 \\
3 & 8 & 8 & 0.7 & 380 & 480 \\
4 & 6 & 5 & 0.6 & 660 & 1280 \\
5 & 5 & 3 & 0.6 & 1250 & 2120 \\
6 & 5 & 3 & 0.7 & 1920 & 3640 \\
\hline
\end{tabular}

Table 3 Tension tests data

\begin{tabular}{llllll}
\hline ID\# & D & L & R & Disc thickness & avg. eng. strain rate \\
\hline 1 & 8 & 12 & 2 & - & 0.001 \\
2 & 6 & 6 & 1.5 & - & 0.01 \\
3 & 6 & 9 & 1.5 & 0.4 & 350 \\
4 & 6 & 9 & 1.5 & 0.5 & 450 \\
5 & 6 & 6 & 1.5 & 0.6 & 1050 \\
6 & 6 & 4.5 & 1.5 & 0.6 & 1300 \\
\hline
\end{tabular}

\section{Experimental Test Examples}

Several tension and compression tests were made on aluminum AA6061-T6 specimens to evaluate the apparatus functionality and the obtainable range of strain rate. Cylindrical specimens were used for the compression tests, while the tension tests were conducted on threaded specimens with fillets to reduce the stress triaxiality. To facilitate the comparison and the assessment of the repeatability, all specimens were obtained from the same bar.

It should also be pointed out that the experimental apparatus used is identical for tensile and compression tests, indeed it uses the same loading system and the same strain gauges to measure the stress waves. In tensile tests the specimens are directly screwed into the threaded bar extremities. On the contrary, quenched steel caps are mounted at the specimen/bar interfaces to perform compression tests as shown in Fig. 13.

It was possible to cover a relatively wide range of strain rates by varying the specimen size and the sacrificial disc thickness. The main test data are reported in Tables 2 and 3 for compression and tension tests, respectively. The average strain rates are coherent with the values indicated in Table 1, exception made for the compression test ID6, which was much shorter than the typical specimen length above considered.

The results of the compression tests are reported in Fig. 14a and $\mathrm{b}$ in terms of true stress and true strain rate versus true strain. Figures $15 \mathrm{a}$ and $\mathrm{b}$ refer to the tensile tests. The legends reports the average engineering strain rate for each test, which ranged from slightly more than $140 \mathrm{~s}^{-1}$ up to $1920 \mathrm{~s}^{-1}$ in compression and from 350 to 1300 in tension.

The curves show a moderate increase of the tensile strength with increasing strain rate for the tested AA6061T6 alloy. A noticeable increase in elongation at break is observed, denoting an apparently higher ductility in dynamic conditions. However, the stress triaxiality varies among the different geometries, and this may affect the final elongation.

A complete characterization of the material, including the calibration of a constitutive model, is outside the scope of this paper and is not described here. However, the flow
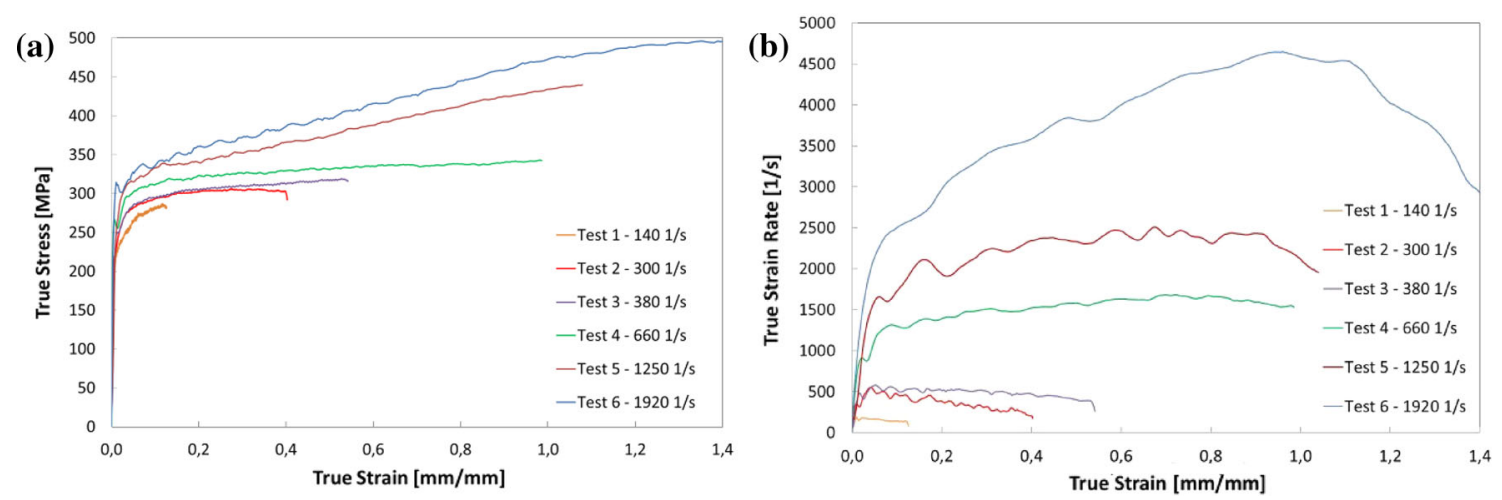

Fig. 14 Compression tests results: a true stress versus true strain, $\mathbf{b}$ true strain rate versus true strain 

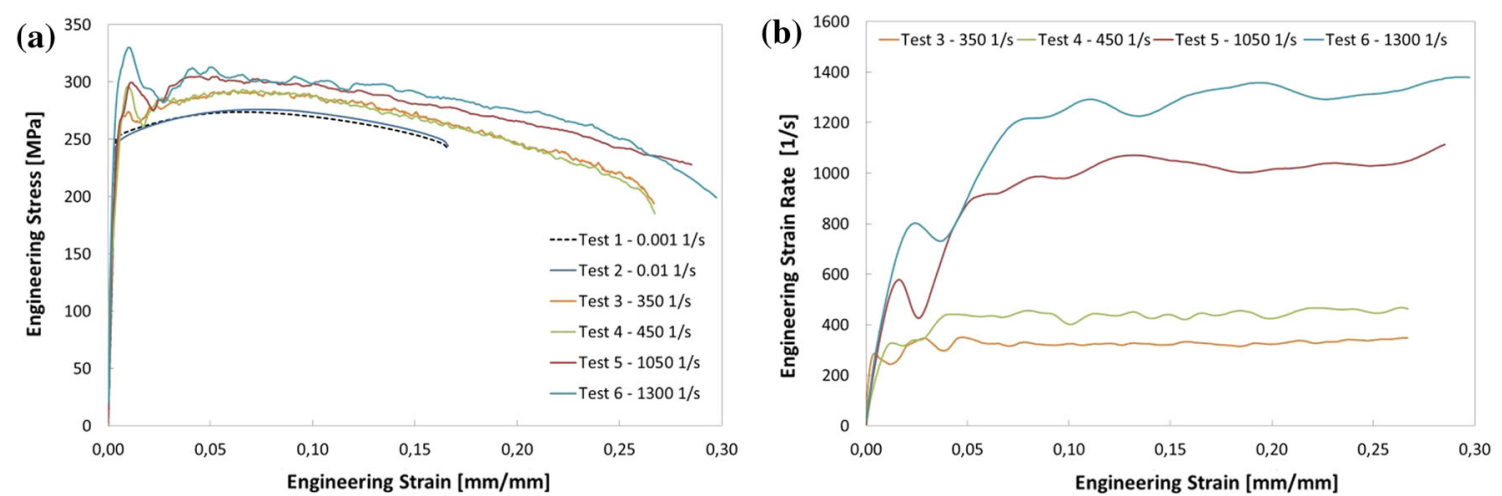

Fig. 15 Tension tests results: a stress versus strain, b strain rate versus strain

stress curves of Figs. 14 and 15 are comparable to those obtained by Owolabi et al. [26]. and by Zhu et al. [27] on the same nominal alloy. Also the strain rate sensitivity is in accordance with previous works [27-29].

Finally, the set-up provides optical access in both compression and tension tests. This could be used to evaluate the deformation process by fast camera [27] or to assist finite element updating procedures for the identification of constitutive model parameters, as done by Sasso et al. [30].

\section{Conclusions}

In this work an innovative wave generation system for SHB in direct tension is demonstrated. The system is based on the shear rupture of a thin brittle disc. It allows direct compression and tension tests to be performed by just inverting the direction of the actuation force. The fixture connecting the actuator to the pre-stressed bar accomplishes the double task of transmitting the preload and breaking the sacrificial element. The apparatus was designed to get an extremely rapid release of the pre-stressing load, using both upstream and downstream configurations. In this way, the apparatus and instrumentation for tension and compression tests are identical. Moreover, the rise time of the input wave is close to the typical values obtained with the classical striker bar-based version of the Hopkinson bar. Tension and compression tests were conducted on AA6061-T6 alloy samples to demonstrate the capability of the equipment.

\section{References}

1. Song B, Chen W (2010) Split Hopkinson Kolsky bar: design testing and applications. Springer, New York. ISBN 978-1-44197981-0
2. Hopkinson B (1914) A method of measuring the pressure produced in the detonation of high explosives or by the impact of bullets. Philos Trans R Soc Lond A 213:437-456

3. Kolsky H (1949) An investigation of the mechanical properties of materials at very high rates of loading. Proc Phys Soc Lond B 62:676-700

4. Staab GH, Gilat A (1991) A direct-tension split Hopkinson bar for high strain rate testing. Expr Mech 31(3):232-235

5. Lewis JL, Campbell JD (1972) The development and use of a torsional Hopkinson-bar apparatus. Expr Mech 12:520-524

6. Lindholm US, Yeakey LM (1968) High strain-rate testing: tension and compression. Expr Mech 8:1-9

7. Nicholas T (1980) Tensile testing of materials at high strain rate of strain. Expr Mech 21:177-185

8. Bragov AM, Lomunov AK (1995) Methodological aspects of studying dynamic material properties using the Kolsky method. Int J Impact Eng 16:321-330

9. Chen W, Lu F, Cheng M (2002) Tension and compression tests of two polymers under quasi-static and dynamic loading. Polym Test 21(2):113-121

10. Huh H, Kang WJ, Han SS (2002) A tension split Hopkinson bar for investigating the dynamic behavior of sheet metals. Expr Mech 42(1):8-17

11. Yokoyama T (2003) Impact tensile stress-strain characteristics of wrought magnesium alloys. Strain 39(4):167-175

12. Smerd R, Winkler S, Salisbury C, Worswick M, Lloyd D, Finn M (2005) High strain rate tensile testing of automotive aluminum alloy sheet. Int J Impact Eng 32(1e4):541-560

13. Taniguchi N, Nishiwaki T, Kawada H (2007) Tensile strength of unidirectional CFRP laminate under high strain rate. Adv Compos Mater 16(2):167-180

14. Nie X, Song B, Ge Y, Chen WW, Weerasooriya T (2009) Dynamic tensile testing of soft materials. Expr Mech 49(4):451-458

15. Robert G, Christian K, Nik P (2012) A new split Hopkinson tensile bar design. Int J Impact Eng 50:63-67

16. Albertini C, Montagnani M (1974) Testing techniques based on the split Hopkinson bar. Institute of Physics, London, Conf. Ser. No. 21:22-31

17. Albertini C, Labibes K, Split Hopkinson bar testing apparatus. WIPO, International Patent WO 98/26274

18. Albertini C, Cadoni E, Labibes K (1998) Mechanical characterization and fracture process of concrete at high strain-rates. In: Gjørv, Sakai, Banthia (eds) Concrete under severe conditions, vol 2. E\&FN Spon, London, pp 735-744

19. Cadoni E, Albertini C, Solomos G (2006) Analysis of the concrete behavior in tension at high strain-rate by a modified Hopkinson bar in support of impact resistant structural design. J Phys IV 3(9):647-652 
20. Mirone G (2013) Development of a shape memory alloy: activated clamping, device for split Hopkinson tension bars. Exp Tech. doi:10.1111/ext.12008

21. Quik M, Labibes K, Albertini C, Valentin T, Magain P (1997) Dynamic mechanical properties of automotive thin sheet steel in tension, compression and shear. J Phys IV 7:C3-C379

22. Tarigopula V, Hopperstad OS, Langseth M, Clausen AH, Hild F (2008) A study of localization in dual-phase high-strength steels under dynamic loading using digital image correlation and FE analysis. Int J Solids Struct 45:601-619

23. Chen Y, Clausen AH, Hopperstad OS, Langseth M (2011) Application of a split-Hopkinson tension bar in a mutual assessment of experimental tests and numerical predictions. Int J Impact Eng 38(10):824-836

24. Davie EDH, Hunter SC (1963) The dynamic compression testing of solids by the method of the split Hopkinson pressure bar. J Mech Phys Solids 11(3):155-179

25. Sasso M, Newaz G, Amodio D (2008) Materials characterization at high strain rate by Hopkinson bar tests and finite element optimization. Mater Sci Eng 487:289-300
26. Owolabi G, Odoh D, Peterson A, Odeshi A, Whitworth H (2013) Measurement of the deformation of aluminum alloys under high strain rates using high speed digital cameras. World J Mech 3:112-121

27. Zhu D, Mobasher B, Rajan SD, Peralta P (2011) Characterization of dynamic tensile testing using aluminum alloy 6061-T6 at intermediates strain rates. J Eng Mech 137:669-679

28. Manes A, Peroni L, Scapin M, Giglio M (2011) Analysis of strain rate behavior of an Al 6061 T6 alloy. Procedia Eng 10:34773482

29. Leuser DR, Kay GJ, LeBlanc MM (1999) Modeling large-strain, high-rate deformation in metals, 3th Biennal Tri-Lab. Eng. Conference on Modeling and Simulation, Pleasaton

30. Sasso M, Fardmoshiri M, Mancini E, Rossi M, Cortese L (2015) High speed imaging for material parameters calibration at high strain rate. Dymat, Lugano 